ATUALIZAÇÃO

\title{
MODELO PRODUTIVO
}

\section{PARAA LEPTOSPIROSE}

Denise Santos Correia de Oliveira, ${ }^{1}$ Maria José Bezerra Guimarães ${ }^{2}$ e Zulma Medeiros ${ }^{3}$

\section{RESUMO}

A incidência da Leptospirose em países da América Latina tem crescido associada ao crescimento desordenado das cidades e a desastres naturais. No Brasil, ocorreram cerca de 9.447 casos de leptospirose com 1.132 óbitos entre 2003 e 2005. A letalidade média registrada no país é de 12\%. A doença apresenta diversidade de modos de transmissão e disseminação, mas os roedores são considerados o principal reservatório da doença. As precárias condições de vida no ambiente urbano contribuem para um convívio humano muito próximo desses reservatórios, expondo as populações ao risco de contaminação. Neste artigo, sistematizou-se um modelo produtivo para a leptospirose, considerando os determinantes sociais, demográficos, biológicos e climáticos envolvidos na gênese da doença e os desafios para o seu controle.

DESCRITORES: Leptospirose. Zoonose. Saúde Pública.

\section{INTRODUÇÃO}

Aleptospirose é uma zoonose de distribuição mundial que tem como agente etiológico uma bactéria helicoidal (espiroqueta) do gênero Leptospira. Este gênero possui duas espécies, segundo a classificação taxonômica clássica: L. interrogans, que compreende todas as leptospiras patogênicas, e L. biflexa, que corresponde às leptospiras saprófitas isoladas no ambiente. Ambas são divididas em diversas

1 Secretaria de Saúde do Recife e Centro de Pesquisas Aggeu Magalhães, Fundação Oswaldo Cruz (CPqAM - FIOCRUZ).

2 Secretaria de Saúde do Recife.

3 Centro de Pesquisas Aggeu Magalhães, Fundação Oswaldo Cruz (CPqAM, FIOCRUZ) e Instituto de Ciências Biológicas, Universidade de Pernambuco (ICB-UPE).

Endereço para correspondência: Zulma Medeiros, Laboratório de Doenças Transmissíveis, Departamento de Parasitologia, Centro de Pesquisas Aggeu Magalhães, Av. Moraes Rego, s/n. Recife 50670-420, Pernambuco, Brasil. E-mails: medeiros@cpqam.fiocruz.br ,denise.oliveira@recife.pe.gov. br ou odenise@terra.com.br.

Recebido para publicação em: 19/6/2008. Revisto em: 24/12/2008. Aceito em: 10/3/2009. 
sorovariedades (sorovares), que estão agrupadas em sorogrupos antigenicamente semelhantes (Vasconcellos, 2000; Levett, 2001). Contudo, o desenvolvimento da biologia molecular tem proposto mudanças taxonômicas, classificando em três espécies não patogênicas $-L$. biflexa, $L$. meyerii, $L$. wolbachii - e sete patogênicas - L. interrogans, L. inadai, L. kirschneri, L. noguchii, L. santarosai, $L$. weilii e $L$. borgpetersenii (Zunino e Pizarro, 2007).

O homem é considerado um hospedeiro acidental e terminal dentro da cadeia de transmissão, sendo pouco eficiente na transmissão da doença. A leptospirose possui diversas formas clínicas, que vão desde a infecção subclínica (podendo apresentar doença febril anictérica com ou sem meningite, uveítes) até a forma grave com elevada letalidade, com icterícia, insuficiência renal e hemorragia (doença de Weil) (Sakata et al., 1992; Sanders et al., 1999; Levett, 2001; Costa et al., 2001; Zunino e Pizarro, 2007).

\section{FONTES DE INFECÇÃO DA DOENÇA}

A leptospirose é uma antropozoonose que tem os animais silvestres, sinantrópicos e domésticos, como seus hospedeiros primários. Vasconcelos (2000) classifica a doença segundo três ambientes básicos: rural, silvestre e urbano. De acordo com os grupos de animais e as variáveis ambientais, a doença manifesta-se na população animal sob a forma de surtos epidêmicos ou permanece dentro de um limite de endemicidade.

Os roedores são considerados os principais reservatórios da doença, sendo-lhes atribuída a maior responsabilidade pela sua transmissão. Importância especial deve ser conferida à espécie Rattus norvegicus, principal espécie transmissora em centros urbanos, conhecida como ratazana de esgotos. Sua proliferação é verificada em grandes cidades, onde as redes pluvial e de esgotos não recebem tratamento adequado e, com freqüência, se interconectam possibilitando uma maior contaminação ambiental (Possas 2000; Sarkar et al., 2002). Contudo, outros animais, especialmente os cães, participam da cadeia de transmissão da doença. Estes, quando infectados, podem eliminar leptospiras por meio da urina, durante meses, sem apresentar sintomas (Freitas et al., 2004; Blazius et al., 2005; Batista et al., 2005; Brown et al., 2008).

Em áreas rurais, o cão exerce também um importante papel na cadeia de transmissão. Mas, outros animais de produção, como bovinos, suínos, equinos, bubalinos, caprinos e ovinos, estão susceptíveis à infecção e participam da transmissão da doença por meio do contato dessas espécies com trabalhadores do setor agropecuário (Vasconcellos, 2000; Homem et al., 2001).

Quanto ao ambiente silvestre, estudos têm mostrado diversos animais selvagens com anticorpos positivos para a doença, o que contribui para a permanência da infecção neste ciclo (Souza Júnior et al., 2006; Jansen et al., 2007). Há relatos de casos de leptospirose humana contraída neste ambiente, associados com atividades de recreação em águas naturais que são utilizadas por animais silvestres e de produção 
pecuária (Lima et al., 1990; Sarkar et al., 2002; Tassinari et al., 2004; Jansen et al; 2007; Brown et al, 2008). Observa-se uma estreita relação entre determinados sorovares e algumas espécies animais. A investigação para identificar o sorovar circulante pode indicar a provável fonte de infecção entre os animais (Pereira, 2005).

\section{ASPECTOS EPIDEMIOLÓGICOS DA LEPTOSPIROSE}

Apesar da ampla distribuição mundial, a leptospirose é mais frequente em região tropical do que em região de clima temperado (Levett, 2001; Brown et al., 2008). Isto ocorre, sobretudo, em razão das condições ambientais de calor e umidade que favorecem a manutenção da bactéria no meio ambiente. A doença é sazonal nos países de clima tropical com epidemias observadas em estações chuvosas ou após desastres naturais (Sanders et al., 1999; Ko et al., 1999; Costa et al, 2001; Sarkar et al., 2002; Tassinari et al., 2004).

Nos últimos anos, constatou-se aumento das notificações da doença em vários países do mundo: Nicarágua, Austrália, Estados Unidos, Índia, Sudeste da Ásia, Malásia e Brasil. No mundo, cerca de dez mil casos são notificados por ano em todas as grandes metrópoles, sendo uma das epidemias urbanas frequentes na América Latina (Levett, 2001; Tassinari et al., 2004; Mcbride et al; 2005).

Epidemias e surtos associados à doença febril com hemorragia pulmonar e alta letalidade têm sido relatados (Zaki e Shieh, 1996). A letalidade depende, entre outros fatores, dos sorovares infectantes, da gravidade da forma clínica, da precocidade do diagnóstico, do tratamento prescrito e da faixa etária do paciente. As formas graves da doença produzem uma taxa de letalidade que pode variar entre 5\% e 40\% (Zunino e Pizarro, 2007).

Existem relatos da doença como de risco ocupacional, sendo observada em trabalhadores de arrozais, canaviais, minas, abatedouros, tratadores de animais, fazendeiros e médicos veterinários, com prevalência variando entre $10,4 \%$ e $28,5 \%$ (Almeida et al., 1999). Inquéritos sorológicos para a detecção de anticorpos têm sido realizados em trabalhadores de serviços de saneamento (rede de abastecimento de água, esgotos, coletadores de lixo e varredores) com a prevalência variando de 7,6\% a 16,7\% (Almeida et al., 1994; Almeida et al., 1999; Gonçalves et al., 2006). Contudo, Everard, Hayes e Fransechanpong (1985) encontraram dificuldade em relacionar a doença ao risco ocupacional, recomendando ser necessário considerar as características ambientais das localidades de residência das populações estudadas. Almeida e colaboradores (1999), ao realizarem um inquérito sorológico entre os trabalhadores do serviço de saneamento ambiental em Pelotas (RS), reforçaram esta recomendação.

Reis e colaboradores (2008) encontraram a prevalência de $15,4 \%$ em uma localidade de baixa condição de vida na cidade de Salvador, sendo observada elevada prevalência entre adolescentes e adultos. O risco maior de adquirir a doença ficou evidenciado entre as pessoas que residiam a menos de 20 metros de esgoto aberto, confirmando a importância da localização da residência em áreas com precárias condições de saneamento. 
Nos centros urbanos, o crescimento desordenado e a produção de lixo propiciam condições ambientais favoráveis para a reprodução da população de roedores, principais reservatórios da doença (Figueiredo et al., 2001; Tassinari et al., 2004).

Como fator potencializador, fortes chuvas sazonais em regiões tropicais, ao provocarem enchentes e inundações, podem ocasionar epidemias de leptospirose em áreas urbanas, constituindo uma condição favorável para a exposição ao agente da doença por meio da transmissão hídrica (Barcellos e Sabroza, 2001; Costa et al., 2001; Tassinari et al., 2004). As alterações ambientais resultantes da destruição das áreas ribeirinhas modificam o curso natural e o fluxo dos rios, facilitando maior extensão das inundações, o que expõe um número maior de pessoas à doença. Outro fator importante é a altitude, pois áreas mais baixas podem concentrar coleções de água ou interferir no curso das águas de enchentes e de desastres naturais (Barcellos e Sabroza, 2001; Barcellos et al., 2003). Entretanto, Pires (2006) defende a hipótese de que, nos períodos das enchentes, há uma maior sensibilidade para o diagnóstico da doença. Isso poderia criar um artifício em relacionar a elevada detecção de casos com alta pluviosidade.

No Brasil, os primeiros registros de leptospirose ocorreram nas décadas de 1910 e 1940, sendo inicialmente confundida com febre amarela. Infecções e formas graves da doença foram reconhecidas na década de 1930. Posteriormente, na década de 1960, vários surtos urbanos foram relatados nas grandes cidades brasileiras (Gonçalves et al., 2006).

No período de 1985 a 1997 foram notificados, no Brasil, 35.403 casos da doença, variando entre 1.594 e 5.576 casos anuais, com um total de 3.821 óbitos registrados (Brasil, 2003). Entre 1999 e 2003, foram confirmados 14.334 casos com uma média anual de 2.866 casos e 336 óbitos. De 2003 a 2005 foram confirmados 1.132 óbitos e 9.447 casos, com maior ocorrência entre o grupo etário de 20 a 49 anos. O coeficiente de incidência no país foi de 1,7/100.000 habitantes e a letalidade média, de 12\% (Brasil, 2005a). A Região Sul detém o maior coeficiente de incidência e a Região Centro-Oeste, o menor (0,5/100.000 habitantes) (Brasil, 2006). Pereira et al. (2000) relatam que Leptospira interrogans sorovar Copenhageni é o principal agente etiológico da doença em humanos nas Regiões Sudeste e Nordeste do Brasil, sendo ainda escassas as informações nas demais regiões do país.

Entre 1991 e 1999, ocorreram 32.350 internações hospitalares por leptospirose, com uma média de 3.600 internações ao ano. As internações ocorrem nas formas mais graves da doença, que representam cerca de 5\% a $40 \%$ de todas as formas clínicas. Estes dados mostram que o real número de casos associados às epidemias pode ser superior aos detectados pelos serviços de vigilância epidemiológica (Possas 2000; Silva et al., 2003, Zunino e Pizarro, 2007).

Um dos fatores importantes para a diminuição da letalidade das formas graves é o diagnóstico precoce. Ko e colaboradores (1999) descrevem uma epidemia na cidade de Salvador com elevada letalidade e chamam a atenção para a dificuldade de se estabelecer um diagnóstico diferencial com dengue no início dos sintomas. É 
provável que mais óbitos ocorram, devido às epidemias de dengue e leptospirose que ocorrem simultaneamente em países de clima tropical como o Brasil (Sanders et al., 1999; Sarkar et al; 2002).

\section{FATORES DETERMINANTES DO MODELO PRODUTIVO DA LEPTOSPIROSE}

Toda infecção implica a interação de componentes essenciais ligados à fonte, ao hospedeiro e ao ambiente. $\mathrm{O}$ reservatório pode ser influenciado pela criação e instalação de um ambiente antrópico favorável que pode provocar mudanças em seu comportamento. A domiciliação ou a sinantropia de muitas populações dentro de um sistema ecológico é um exemplo deste fato. As espécies de roedores apresentam um potencial de adaptação importante, tendo sido dispersas no mundo por meio da ação antrópica (Forattini, 2004; Priotto e Steimann, 2003).

O grau de convivência das populações sinantrópicas com a população humana tem como tradução a intensidade e a frequência do aparecimento de doenças transmissíveis a elas associadas. Quanto às espécies onívoras, por encontrarem recursos mais fartos nas áreas habitadas do que em seu meio natural, podem atingir densidades consideráveis na natureza adaptando-se ao espaço, e isso não ocorre sem risco de desequilíbrio entre as populações que convivem em um mesmo espaço (Forattini, 2004).

É também importante conhecer, no processo de determinação das doenças, o papel dos fatores demográficos, sociais e econômicos (Teixeira et al., 1999). Os fatores de risco associados à transmissão da leptospirose dependem de características da organização espacial e das condições de vida e de trabalho da população (Barcellos et al., 2003).

De acordo com a organização social, criam-se diferentes graus de distribuição dos recursos produzidos, resultando em desigualdades socioeconômicas e da qualidade de vida das populações. Características do ambiente social reforçam as doenças de uma maneira geral, seja na produção da doença ou na recuperação da saúde (Contandiopoulos, 1998).

Considerando ainda a influência dos fatores ambientais na produção da doença, a variabilidade climática é outro componente que afeta a saúde humana, principalmente por alterações nos padrões de determinadas doenças infecciosas, criando condições ambientais mais favoráveis para a reprodução e a sobrevivência de patógenos e vetores. Fatores como temperatura, umidade relativa, precipitação pluviométrica e até o ciclo hidrológico poderão afetar a capacidade de reprodução e sobrevivência de patógenos. O clima é considerado um dos determinantes no modelo de produção de algumas doenças infecciosas como malária, dengue, leptospirose, peste bubônica, cólera e arboviroses. A variabilidade climática pode acelerar os ciclos de transmissão, bem como estender as suas áreas de distribuição geográfica, tanto para latitudes quanto para altitudes maiores, incluindo aqui os eventos 
extremos (desastres naturais) tais como: inundações, ciclones, furacões e ondas de calor (Epstein, 1995; Epstein et al., 1995; Confalonieri, 2002; Confalonieri, 2003).

Alguns tipos de eventos meteorológicos extremos contribuem para as epidemias de doenças infecciosas, conforme já foi observado em várias aglomerações urbanas brasileiras durante as chuvas de verão. A leptospirose é um exemplo de doença de veiculação hídrica em desastres naturais (Barcellos et al, 2001; Confalonieri, 2003).

Para a compreensão da leptospirose é necessário considerar os fatores envolvidos na sua cadeia produtiva. $\mathrm{O}$ modelo de produção da doença desenhado na Figura 1 destaca os determinantes relacionados com o reservatório e o ambiente, os fatores demográficos, socioeconômicos e biológicos que devem ser considerados na gênese da leptospirose.

Leptospirose

\begin{tabular}{|c|c|}
\hline \multicolumn{2}{|c|}{ Fatores do reservatório } \\
\hline $\begin{array}{l}\text { Fauna de roedores } \\
\text { - Animais domésticos } \\
\text { - Animais de produção } \\
\text { - Animais silvestres }\end{array}$ & $\begin{array}{l}\text { Características dos roedores } \\
\text { - Taxa de reprodução } \\
\text { - Densidade } \\
\text { - Dispersão } \\
\text { - Alimentação } \\
\text { - Abrigo reprodutivo }\end{array}$ \\
\hline \multicolumn{2}{|c|}{ Fatores ambientais } \\
\hline $\begin{array}{l}\text { - Hidrografia } \\
\text { - Topografia } \\
\text { - Temperatura } \\
\text { - Umidade } \\
\text { - Pluviosidade }\end{array}$ & $\begin{array}{l}\text { - Tipo de solo } \\
\text { Desastres Naturais: inundações e } \\
\text { enchentes, terremotos, furacões, } \\
\text { ciclones. }\end{array}$ \\
\hline Fatores sócio-demográficos & Outros fatores \\
\hline $\begin{array}{l}\text { - Densidade populacional } \\
\text { - Renda } \\
\text { - Escolaridade } \\
\text { - Ocupação } \\
\text { - Moradia }\end{array}$ & $\begin{array}{l}\text { - Número de expostos } \\
\text { - Tempo e repetição de exposição } \\
\text { - Susceptibilidade } \\
\text { - Resposta imunológica }\end{array}$ \\
\hline
\end{tabular}

Figura 1. Modelo produtivo da leptospirose.

\section{PREVENÇÃO E CONTROLE DA LEPTOSPIROSE}

A imunização por meio de vacinas é uma medida de prevenção possível tanto para a população humana quanto para os reservatórios que transmitem a leptospirose ao homem (Levett, 2001). Existem vacinas disponíveis para uso em animais domésticos (cães, bovinos e suínos). As vacinas veterinárias evitam o 
adoecimento do animal, mas não impedem a infecção e necessitam de reforço anual. Os animais imunizados podem eliminar a Leptospira pela urina, tornando-se fonte ativa de transmissão, porém em um período de transmissão menor do que nos animais com a infecção natural (Brasil, 2005; Mcbride et al., 2005).

Para uso humano, Cuba e China desenvolveram vacinas que mostraram eficácia de $78 \%$ e $75 \%$, respectivamente, prevenindo a manifestação clínica da doença por um período de um ano. Estas vacinas não conferem imunidade cruzada e não apresentam imunidade duradoura, são específicas para os sorogrupos que as compõem (Mcbride et al., 2005).

No Brasil, não existe ainda vacina disponível para uso em populações humanas. No entanto, instituições de excelência no país têm desenvolvido estudos com o objetivo de produzir uma vacina de DNA recombinante a partir de proteínas capazes de conferir imunidade (Fiocruz, 2007).

As medidas de controle para a leptospirose devem considerar todos os determinantes na produção da doença (Figura 1). Em relação aos roedores, tem-se adotado o conceito de manejo integrado, que foi originalmente criado para combater pragas da lavoura e depois adaptado ao combate das pragas urbanas, incluindo roedores sinantrópicos. Manejo integrado é um termo abrangente que envolve um conjunto de ações preventivas, corretivas e de eliminação, devendo ser conduzidas, de forma a diminuir os custos e minimizar os riscos envolvidos para a biodiversidade, especialmente para o homem (Brasil, 2002).

Todas as espécies de organismos vivos possuem uma habilidade inerente para a reprodução que garante a perpetuação da espécie. A população de uma determinada espécie, em dado lugar e momento, é resultado da interação da capacidade reprodutiva versus fatores controladores que limitam esta capacidade reprodutiva. Abrigo, água e alimento são fatores essenciais para a sobrevivência da população de roedores. A limitação desses fatores exerce a força de fator controlador da população. Medidas de infraestrutura, como o saneamento, exercem força de fator controlador (Brasil, 2002).

Quanto aos desastres naturais, a quimioprofilaxia é uma medida específica para grupos específicos em risco, como militares, bombeiros e outros, não sendo recomendada sua utilização em grande escala em populações expostas ao risco. A droga mais utilizada é a doxycyclina durante o período de intensa exposição por período específico (Chin, 2001; Mcbride et al., 2005).

O Ministério da Saúde (MS) vem adotando, como medida de prevenção desde 2004, o tratamento pós-exposição em pessoas com síndrome febril após desastres naturais (enchentes e inundações), procurando, com este tratamento precoce, evitar as formas graves da doença (Brasil, 2005b).

\section{COMENTÁRIOS FINAIS}

O conhecimento biológico da doença não é suficiente para entender seu modelo produtivo. A aplicação da biologia molecular tem ampliado o conhecimento 
das espécies patógenas existentes, favorecendo o desenvolvimento de estudos para a busca de vacinas capazes de promover a imunização e o desenvolvimento de tecnologias para o diagnóstico da doença. Contudo, compreender o modelo produtivo da leptospirose não requer apenas o entendimento dos determinantes biológicos, mas também conhecimento da multiplicidade dos determinantes sociais, econômicos, ambientais e climáticos envolvidos no aparecimento da doença, em uma determinada população, em um determinado espaço geográfico.

Deve-se, ainda, ressaltar a necessidade de promover ações para reduzir a letalidade observada no país. A abordagem sindrômica no diagnóstico deve ser estimulada e introduzido na atenção primária o tratamento precoce em síndrome febril pós-enchentes.

A leptospirose é considerada uma doença negligenciada. Não é possível a eliminação do transmissor da doença, nem tampouco evitar os elevados índices de pluviosidade nas regiões de clima tropical, mas é possível estabelecer barreiras para o contato entre o reservatório e o homem e realizar o tratamento precoce nos casos suspeitos para evitar a letalidade indesejada.

O saneamento ambiental, a educação, a informação e a comunicação permanecem sendo os pilares fundamentais para minimizar os danos causados pela doença à população das cidades que contabilizam grande número de casos e óbitos anualmente.

\section{ABSTRACT}

Productive model for leptospirosis

The incidence of leptospirosis in Latin American countries has increased along with disorderly growth of cities and occurrence of natural disasters. In Brazil, around 9,447 leptospirosis cases and 1,132 deaths from this cause occurred between 2003 and 2005. The mean lethality rate recorded in this country is $12 \%$. The disease presents a variety of transmission and dissemination routes, among which rodents are considered to be its main reservoir. Precarious living conditions in urban environments and domiciles in close proximity to these reservoirs contribute towards the population's exposure. This paper systematizes a production model for leptospirosis, taking into account the social, demographic, biological and climatic determinants involved in the genesis of this disease and the challenges in its control.

KEY WORDS: Leptospirosis. Zoonosis. Public health.

\section{REFERÊNCIAS}

1. Almeida LP, Martins LFS, Brod CS, Germano PML, Levantamento soroepidemiológico de Leptospirose em Trabalhadores do serviço de saneamento ambiental em localidade urbana da região Sul do Brasil. Rev Saúde Públ 28: 76-81, 1994. 
2. Almeida LP, Martins LFS, Brod CS. Fatores de risco associados à presença de anticorpos antileptospira em trabalhadores do serviço de saneamento ambiental. Ciência Rural 29: 511-516, 1999.

3. Barcellos C, Sabroza PC. The place behind the case: leptopirosis risks and associated environmental conditions in a flood-related outbreak in Rio de Janeiro. Cad Saúde Públ 7: 59-67, 2001.

4. Barcellos C, Lammerhirt CB, Almeida MAB, Santos E. Distribuição espacial da Leptospirose no Rio Grande do Sul, Brasil. Recuperando a ecologia dos estados ecológicos. Cad Saúde Públ 19: 1283-1292, 2003.

5. Batista CSA, Alves CJ, Vasconcellos SA, Morais ZM, Clementino IJ, Alves FAL, Lima FS, Araújo Neto JO. Soroprevalência e fatores de risco para leptospirose em cães de Campina Grande, Paraíba. Arq Bras Med Vet 57: 179-185, 2005.

6. Blazius DR, Romão PRT, Blaziuas EMC, Silva OS. Ocorrência de cães errantes soropositivos para Leptospira spp na cidade de Itapema, Santa Catarina, Brasil. Cad Saúde Públ 21: 1952-1956, 2005.

7. Brasil, Ministério da Saúde. Manual de controle de roedores. Ministério da saúde. Fundação Nacional de Saúde. Brasília, 2002.

8. Brasil, Ministério da Saúde. Guia de Vigilância Epidemiológica. Brasília. Ministério da Saúde. 2003.

9. Brasil, Ministério da Saúde. Guia de Vigilância Epidemiológica. Brasília. Ministério da Saúde, 2005a. p. 67-83.

10. Brasil, Ministério da Saúde. Vigilância em Saúde no SUS. Ministério da Saúde. Brasília, 2006. p. 196-197.

11. Brasil, Ministério da Saúde. Secretaria de Vigilância à Saúde (SVS/MS). 'Plano de contigência de Vigilância em saúde frente a inundações, 2005b. disponível em: http://portal.saude.gov.br.

12. Brown K, Prescott J Leptospirosis in the family dog: a public health perspective. Can Med Assoc 178: 339-401, 2008.

13. Chin J. El control de las enfermedades transmisibles. Washington, DC. Organización Panamericana de Salud, 2001.

14. Confalonieri UEC, Chame M, Najar A, Chaves SAM, Krug T; Nobre C; Miguez, JDG.; Cortesão J; Hacon S. Mudança Global e Desenvolvimento: Importância para Saúde. Inf Epidemiol SUS 11: 39-154 2002.

15. Confalonieri UEC. Variabilidade climática, vulnerabilidade social e saúde no Brasil. Terra Livre 1, 193-204, 2003. Disponível em: http://www.unit.br/mestrado/saudeambiente/leitura.

16. Contandriopoulos AP. Pode-se construir modelos baseados na relação entre contextos sociais e saúde? Cad Saúde Públ 14: 199-204,1998.

17. Costa E, Costa YA.; Lopes AA.; Sacramento E, Bina JC. Formas graves de leptospirose: aspectos clínicos, demográficos e ambientais. Rev Soc Bras Med Trop 34: 261-267, 2001.

18. Epstein PR. Emergency Diseases and Ecosystem Instability: New threats to public health. Am JPubl Health 85: 168-1970, 1995a.

19. Epstein PR, Pena OC, Racedo JB. Climate and disease in Colombia. The Lancet 346: 1243-1244, 1995 b.

20. Everard COR, Hayes RJ, Fraserchanpong GMA. Serosurvey for leptospirosis in Trinidad among urban end rural dwellers and persons occupationally at risk. Trans Royal Soc Trop Med Hyg 79: 96-105, 1985.

21. Figueiredo CM, Morais AC, Oliveira MAA, Alves WR, Coteman MC, Chamone CBE, Kairy MC. Leptospirose humana no município de Belo Horizonte Minas Gerais, Brasil: uma abordagem geográfica. Rev Soc Bras Med Trop 34: 331-338, 2001.

22. Fundação Oswaldo Cruz (Fiocruz), Bio-manguinhos. Informe de Bio-manguinhos Disponível em www.bio.fiocruz.br/interna/2004_03_diariopopularRS_leptospirose.htm

23. Forattini OP. Ecologia, Epidemiologia e Sociedade. São Paulo: Artes Médicas 2a ed, 2004.

24. Freitas JC, Silva FG, Oliveira RC, Delbem ACB, Muller EE, Alves LA, Teles PS. Isolation of leptospira spp from dogs, bovine and swine naturally infected. Ciência Rural 34: 853-856, 2004.

25. Gonçalves DD, Teles PS, Reis CR, Lopes FMR, Freira RL, Navarro IT, Alves LA, Muller EE, Freitas JC. Seroepidemiology and occupational and environmental variables for leptospirosis, brucellosis and toxoplasmosis in slaughterhouse workers in the Paraná state, Brazil. Rev Inst Med Trop São Paulo 48: 135-140, 2006. 
26. Homem VSF, Heinemann MB, Moraes ZMM, Vasconcellos SAF, Ferreira F, Ferreira Neto, JS. Estudo epidemiológico da leptospirose bovina e humana na Amazônia oriental brasileira. Rev Soc Bras Med Trop 34: 173-180, 2001.

27. Jansen A, Luge E, Guerra B, Wittschen P, Gruber AC, Loddenkemper C, Schneider, T, Lierz, M, Ehlert, D, Appel, B, Stark, K, Nockerlt, K. Leptospirosis in urban Wild Boars, Berlin, Germany. Em Infect Dis 13: 739-742, 2007.

28. Ko AI, Reis MG, Dourado CMR., Johnson Jr W, Riley LW. Urban Epidemic of severe leptospirosis em Brasil. Lancet 354: 880-825, 1999.

29. Levett PN. Leptospirose. Clin Microbiol Rev 14: 296-326, 2001.

30. Lima SC, Sakata EE, Santo CER, Yasuda PH, Stiliano SV, Ribeiro FA. Surto de leptospirose humana por atividade recreacional no município de São José dos Campos, São Paulo. Estudo soroepidemiológico. Rev Inst Med Trop São Paulo 32: 474-479, 1990.

31. Mcbride AJA; Athanazio DA, Reis MG, Ko AI. Leptospirosis. Curr Opin Infect Dis 18: 376-386, 2005.

32. Pereira MM. Leptospirose. In: Coura JR. Dinâmica das doenças infecciosas e parasitárias. Editora Guanabara Koogan, 2005. p.1497-1508.

33. Pereira MM, Pereira da Silva JJ, Bauab AR, Vasconcellos AS, Moraes ZM Baranton G, Saint Girous JA. Clonal subpopulation of Leptospira interrogans sensu stricto is the major cause of leptospirosis outbreaks in Brazil. J Clin Microbiol 38: 450-452, 2000.

34. Pires FDA. Leptospirose e enchentes: uma falsa correlação? Rev Patol Trop 35: 199-204, 2006.

35. Possas CA. Urbanização, ecologia e emergência de formas graves da leptospirose: análise comparativa de dados secundários nacionais. In: Anais do evento comemorativo do centenário do Instituto Oswaldo Cruz e da Fundação Oswaldo Cruz. Rio de Janeiro, 2000.

36. Priotto J, Steimann A. Biologia dos Roedores In: Polop J, Priotto J. Steinmann A, Provensal C, Castilho E, Calderón G, Enria D, Sabattini M, Coto H. Manual de control de roedores en municípios. Argentina. Universidad Nacional de Rio Cuarto 11-23. 2003.

37. Reis RB, Ribeiro GS, Felzemburgh RDM, Santan FSS, Mohr S, Melendez AXTO, Queiroz A, Santos AC, Ravines, RR, Tassarine WS, Carvalho MS, Reis, MG, Ko, AI. Impact of environment and social gradient on Leptospira infection in urban slums. Negl Trop Dis 2: 1-10, 2008.

38. Sakata EE, Yasuda PH, Romero EC, Silva MV, Lomar AV. Sorovares de Leptospira interrogans isolados de casos de leptospirose humana em São Paulo. Brasil. Rev Inst Med Trop São Paulo 34: 217-221, 1992.

39. Sanders EJ, Rigan-Perez JG, Smits HL, Deseda CC, Varndam VA, Aye T, Spiegel RA, Weyant RS, Bragg SL. Increase of leptospirosis in dengue -negative patients after a hurricane in Puerto Rico, 1966. Am J Trop Med Hyg 61: 399-404, 1999.

40. Sarkar V, Nascimento SF, Barbosa R, Martins R, Nuevo H, Kalafanos I, Grunstein, I, Flannery B, Dias J, Rirey L, Reis M, Ko AI. Population-Based Case-control investigation of risk factors for leptospirosis during in Urban Epidemic. Am J Trop Med Hyg 66: 605-610, 2002.

41. Silva HR, Tavares-Neto J, Bina JC, Meyer R. Leptopirose-infecção e forma subclínica em crianças de Salvador, Bahia. Rev Soc Bras Med Trop 36: 227-233 2003.

42. Souza Júnior MF, Lobato ZIP, Lobato FCF, Moreira EC,Oliveira RR, Leite GG, freitas TDF, Assis RA. Presença de anticorpos da classe IgM de Leptospira interrogans em animais silvestres do estado de Tocantins. Rev Soc Bras Med Trop 39: 292-294, 2006.

43. Tassinari WS, Pellegrini DCP, Sabroza PC, Carvalho MS. Distribuição espacial da leptospirose no Município do Rio de Janeiro, Brasil ao longo dos anos de 1996 - 1999. Cad Saúde Públ 20: 1721-1729, 2004.

44. Teixeira MG, Barreto ML, Guerra Z. Epidemiologia de prevenção do Dengue. Informe Epidemiológico do SUS 8: 5-33, 1999.

45. Vasconcellos SA Leptospirose em animais domésticos e silvestre Prevenção e controle In: Anais do evento comemorativo do centenário do Instituto Oswaldo Cruz e da Fundação Oswaldo Cruz, Rio de Janeiro, 2000. p. 181-208.

46. Zaki SR, Shieh W J. Leptospirosis associated with outbreak of acute febrile illness and pulmonary haemohage, Nicaragua, 1995. Lancet 347: 53, 1996.

47. Zunino E, Pizarro RP. Leptospirose. Puesta al día. Rev Chil Infect 24 :220-226, 2007. 